\title{
SPECTRAL LUMINESCENCE PROPERTIES OF INDOTRICARBOCYANINE DYE IN BIOLOGICAL TISSUES
}

\author{
M. P. Samtsov, ${ }^{a^{*}}$ E. S. Voropai, ${ }^{b}$ D. G. Mel'nikov, ${ }^{a}$ \\ L. S. Lyashenko, ${ }^{\text {A. A. Lugovskii, }}$ and Yu. P. Istomin ${ }^{c}$
}

UDC 535.37+535.34

\begin{abstract}
We have established that the shape and position of the maximum in the fluorescence spectrum of an indotricarbocyanine dye in tumor and normal tissues in vivo change over time after intravenous injection of the dye. Based on analysis of the spectral properties of the dye in vivo and in blood plasma, the dependence of the properties on the time since injection has shown that in the living body, the environment of the dye molecule changes as the photosensitizer goes from the skin into the tissue. We have established that in tissues in vivo, the dye molecules are localized in a region with low dielectric constant of the medium. We have shown that the change in the ratio of the concentrations of the different forms of hemoglobin in the blood has an effect on the absorption and shape of the fluorescence spectrum of the dye in tissues in vivo.
\end{abstract}

Keywords: indotricarbocyanine dyes, tumor and normal tissues, fluorescence, light absorption by hemoglobin.

Introduction. Polymethine dyes (PDs) are promising for use as photosensitizers for photochemotherapy (also called photodynamic therapy, PDT) [1]. This is first of all due to the presence of absorption bands in their spectra in a range in which biological tissues have greatest transparency (the phototherapeutic window) [2]. For a number of dyes in this class, high photoactivity was found in experiments on cell cultures and also on animals with tumors $[1,3,4]$. The prospects for using a specific photosensitizer mainly depend on how efficiently the molecules in the excited state can generate active intermediates. The probabilities of intramolecular processes for dissipation of the electronic excitation energy for tricarbocyanine dyes depend on the nature of the surrounding molecules [5]. In low-polarity organic solvents, cationic PDs may be found in the form of ions or a mixture of several types of ion pairs [6]. As shown in [7], cationic PDs in a HeLa cell culture are localized in a region with low dielectric constant, and are found mainly in the contact ion pair state. The nature of the environment of the PD molecules in biological tissues in vivo has not been studied to date.

In this paper, we present the results of a study of the spectral luminescence properties of a symmetric indotricarbocyanine dye in a culture of HeLa tumor cells and in tissues in vivo in animals with tumors.

Objects and Methods of Investigation. As the object of investigation, we used an indotricarbocyanine dye synthesized in the spectroscopy laboratory of the A. N. Sevchenko Research Institute of Applied Physical Problems: 2-\{7-[3-methyl-3-ethyl-1-trimethylenecarbo-(6-O-D-glucosyl)-2(1H)-indolenylidene]-4-chloro-3,5-(O-phenyleno)-1,3,5hexatrien-1-yl\}-3-methyl-3-ethyl-1-trimethylenecarbo-(6-O-D-glucosyl)indolenylium bromide (PD1).

In vivo experiments were conducted on mongrel white rats with subcutaneously grafted tumors of the sarcoma 45 (S-45) and sarcoma M1 (SM-1) strains. We used a group of 6-8 animals for the studies. The drug was injected intravenously in the ratio of $1-5 \mathrm{mg}$ per $\mathrm{kg}$ of the animal's weight. The fluorescence spectra of PD1 in vivo was recorded using a spectrometer developed at the A. N. Sevchenko Research Institute of Applied Physical Problems, in which the exciting radiation was delivered to the test object and the fluorescence was delivered to the polychromator by means of an optical fiber. Biological tissues are characterized by nonuniformity of optical properties, associated pri-

\footnotetext{
*To whom correspondence should be addressed.
}

${ }^{\mathrm{a}}$ A. N. Sevchenko Institute of Applied Physical Problems, 7 ul. Kurchatova, Minsk 220064, Belarus; e-mail: samtsov@bsu.by; ${ }^{b}$ Belorussian State University, Minsk; e-mail: voropay@bsu.by; ${ }^{\mathrm{c}}$ N. N. Aleksandrov National Oncology and Medical Radiology Research and Treatment Center, Minsk, Belarus; e-mail: istomin06@mail.ru. Translated from Zhurnal Prikladnoi Spektroskopii, Vol. 77, No. 3, pp. 438-444, May-June, 2010. Original article submitted November 30, 2009. 
marily with the presence of the circulatory system. In order to make sure the detected signal was averaged over the volume of the test specimen, the cross section of the light beam of the exciting radiation on the surface of the tissues was $\sim 5 \mathrm{~mm}$. The fluorescence was detected directly through the skin. In order to take into account the level of scattered light and intrinsic fluorescence of biological tissues, we made control measurements on untreated animals (i.e., with no injection of the photosensitizer). The spectral sensitivity correction factors of the spectrometer were determined by analysis of the fluorescence spectra for tissue samples containing the dye, as recorded using the spectrometer and a Fluorolog spectrofluorimeter calibrated for spectral sensitivity.

The spectral properties of PD1 in vitro were studied on a culture of HeLa tumor cells. The absorption spectra of the dyes in solutions and in tissue samples were recorded using a PV 1251A spectrophotometer, while the fluorescence spectra were recorded using a Fluorolog spectrofluorimeter. The PD1 concentration in the blood of the animals was determined by extracting the PD1 with a $2 \%$ solution of Triton X-100, followed by recording the fluorescence intensity. The optical density of the extract samples was always checked, and a linear relationship between the fluorescence signal and the dye concentration was maintained as needed by diluting the samples.

Results and Discussion. The position of the absorption and emission spectra of PD1 coincides with the transparency window for biological tissues in vivo $(700-900 \mathrm{~nm})$ [2]. This means that in luminescence studies, there is practically no distortion of the spectral characteristics of polymethine dyes from luminescence of the tissues. Thus when using a semiconductor laser with $\lambda=683 \mathrm{~nm}$ in the spectral range 700-900 nm as the exciting light source, no signal appears from photoluminescence of the tumor or thigh muscle tissue of an untreated rat (Fig. 1). Obviously reliable detection of the fluorescence spectra of a photosensitizer becomes problematic against a background of the intrinsic luminescence of biological tissues.

As a result of detailed studies of the fluorescence spectra of PD1 in vivo over a time period after intravenous injection of the photosensitizer, we established that there is a change in the shape and position of the spectra of the dye in tumor and healthy muscle tissues (Fig. 2). Depending on the time elapsed since injection of the photosensitizer, in the spectra recorded in tumor and muscle tissues, the half-width of the bands increases by $2-5 \mathrm{~nm}$ and we observe a long-wavelength shift of the maxima by $2-4 \mathrm{~nm}$. The nature of the changes in the fluorescence spectra of the dye in normal and tumor tissues is similar, but for observation times $>1 \mathrm{~h}$, differences appear in the spectral parameters for these systems. Thus in the spectra of PD1 in the tumor tissue, the band half-width is 1-2 nm greater and its maximum is shifted by $1 \mathrm{~nm}$ toward longer wavelengths compared with the spectra of PD1 in healthy muscle tissue.

The changes over time in the shape and position of the spectra develop over a period of 40-100 min after injection of the drug, where the intensity of the dye fluorescence decreases several-fold (Fig. 3). The change in the fluorescence intensity corresponds to a decrease in the dye concentration in the animals' blood by about an order of magnitude (Fig. 4). We should point out that after the parameters of the PD1 spectra stabilize, for all the concentrations used, the shape and position of the fluorescence spectrum does not change any further over the course of time until the dye is observed in the tissues.

By comparing the fluorescence intensity of PD1 immediately before and after decapitation of the animal and washout of the blood from the tissues, the contribution of the luminescence of the dye in the blood to the spectrum recorded in vivo is estimated (Fig. 5). As we found, $1 \mathrm{~h}$ after injection of the drug, removal of the blood leads to a less than $\sim 10 \%$ decrease in the fluorescence signal of the tissues, which indicates localization of the dye in the tissues.

Recording the fluorescence spectra of the dye in blood plasma samples taken every 15 min after injection of the drug showed that the band maximum is located at $762 \mathrm{~nm}$ and its half-width is $53 \mathrm{~nm}$. The location of the maximum is approximately the same and the half-width of the spectrum of the dye is similar in vivo in the initial part of the observation. In this case, a decrease in the fluorescence intensity of the dye in vivo occurs symbatically with the change in its concentration in the blood samples (Figs. 3 and 4). The established behavior provides a basis for concluding that during the first hour after injection, the photosensitizer is mainly localized in the blood, and then it is redistributed over the tissues. The microenvironment of the dye changes as a result of such a redistribution, which causes the observed changes in the position and shape of its fluorescence spectrum.

In order to determine the nature of the environment of the dye molecules in the tumor tissues in vivo, we compared the spectral properties of PD1 in a number of media. In Fig. 5, we present the absorption spectra of PD1 in a liver tissue sample from an animal and in a culture of HeLa cancer cells. The positions of the absorption band maxima in these media coincide $(\lambda=735 \mathrm{~nm})$, although the half-widths of the spectra are different. According to [8], 


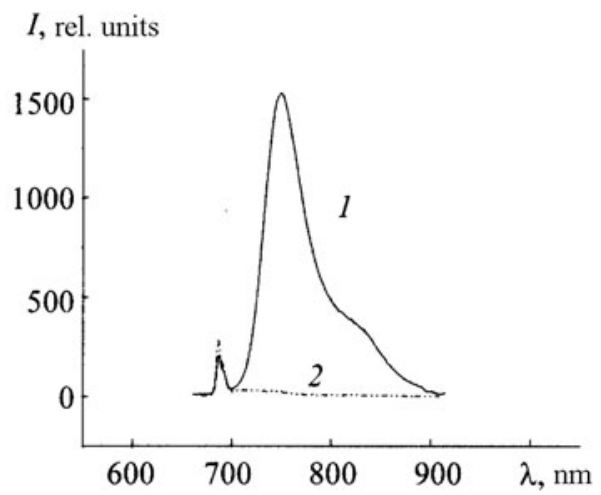

Fig. 1. Fluorescence spectrum of PD1 in vivo (1) and emission spectrum of tumor tissue from untreated rat (2) with excitation at $\lambda=683 \mathrm{~nm}$.

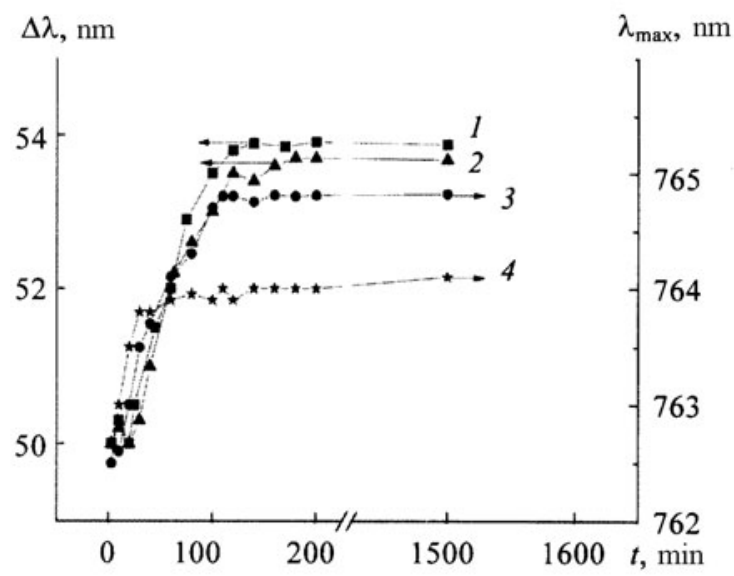

Fig. 2. Half-width $(1,2)$ and position of the maximum $(3,4)$ in the fluorescence spectrum vs. time after injection of PD1 in tumor tissue SM-1 $(1,3)$, in the thigh in vivo $(2,4)$.

the absorption and scattering coefficients for biological tissue in the region of the maximum of the dye absorption spectrum change slightly with wavelength, while in the spectral range corresponding to the half-width of the band, the changes are more significant. Accordingly, scattering and absorption of light by biological tissue may have a certain effect on the half-width of the absorption spectrum of PD1 and, to a lesser degree, may affect the position of its maximum. In this case, the fact that the PD1 absorption band maxima coincide in the liver tissue sample from the animal and in a culture of HeLa tumor cells suggests that the environments of the dye molecules have similar polarities in these systems.

The positions of the maxima in the fluorescence spectra of PD1 coincide for animal tissue samples from which the blood was removed and in a culture of HeLa tumor cells (Fig. 5). In this case, the fluorescence spectrum of PD1 in the tumor tissues in vivo has a smaller half-width $(54 \mathrm{~nm})$ than in the HeLa cells and in the tissue samples from which the blood was removed. In order to determine the reasons for such differences, we should consider that the shape and position of the fluorescence spectrum of the dye in tissues in vivo can be affected by endogenous biomolecules having appreciable absorption in the spectral region of the dye fluorescence [9]. The fluorescence spectra of PD1 overlap the absorption bands of hemoglobin, melanin, and water [10]. The absorption of different forms of hemoglobin is changed to the most significant extent in the fluorescence band of PD1 (710-875 nm): oxyhemoglobin 


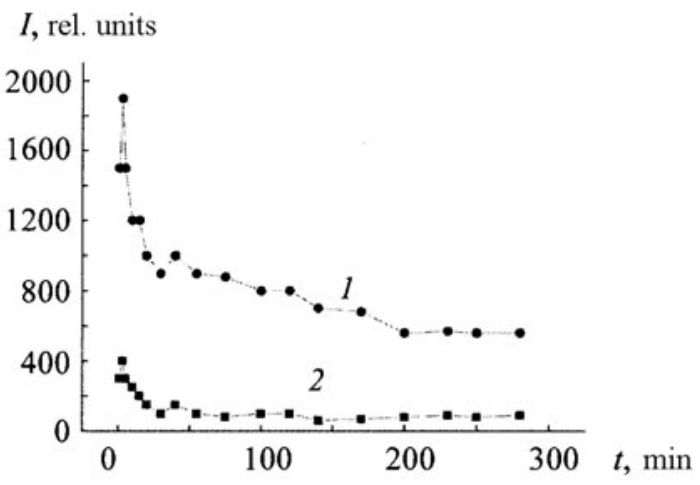

Fig. 3. Pharmacokinetics of accumulation of PD1 in tumor tissue SM-1 (1), in the thigh in vivo (2).

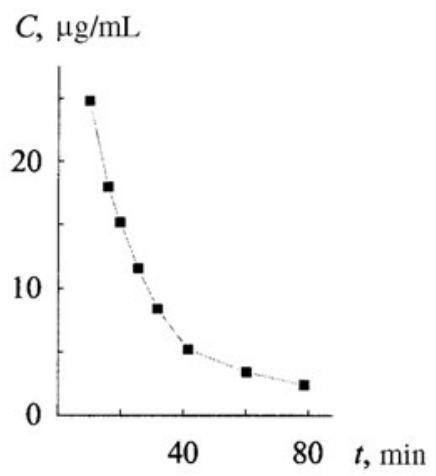

Fig. 4 PD1 concentration in animal blood vs. time after injection.

$\left(\mathrm{HbO}_{2}\right)$, deoxyhemoglobin $(\mathrm{Hb})$, and methemoglobin (MetHb) [10-12]. In HeLa cells, where there is no hemoglobin, and in animal tissue samples from which the blood has been removed (with a significantly lower hemoglobin concentration), the parameters of the fluorescence spectra of the dye are appreciably different from the spectra in tissues in vivo. Taking this into account, the observed differences can be explained from the standpoint of the effect of absorption by hemoglobin of the fluorescence of the dye coming from deep inside the tissues. For this purpose, we carried out a numerical calculation for the fluorescence spectra, taking into account absorption of fluorescence by different forms of hemoglobin.

The absorption spectra of $\mathrm{HbO}_{2}, \mathrm{Hb}$, and MetHb, plotted from the data in [10-13], are shown in Fig. 6. The effect of other existing forms of hemoglobin were not taken into account because their concentration is low and they have weak absorption in the region of the dye fluorescence $[12,13]$. In the calculations, we considered that using the spectrometer, fluorescence of the dye molecules is detected for tumor tissue depth of $\approx 1.5 \mathrm{~cm}$ [14]. In the general case, the expression for the fluorescence spectrum of polymethine dyes in vivo, determined by emission of light by the dye molecules for a biological tissue depth up to $l=1.5 \mathrm{~cm}$, if we take into account absorption by different forms of hemoglobin according to the Bouguer-Lambert-Beer law, can be rewritten as follows:

$$
I(\lambda)=I_{0}(\lambda) \int_{0}^{1.5} 10^{-l\left(C_{\mathrm{HbO}_{2}} \varepsilon_{\mathrm{HbO}_{2}}(\lambda)+C_{\mathrm{Hb}} \varepsilon_{\mathrm{Hb}}(\lambda)+C_{\mathrm{MetHb}} \varepsilon_{\mathrm{MetHb}}(\lambda)\right)} d l,
$$

where $I_{0}(\lambda)$ is the fluorescence spectrum of the polymethine dye molecules emitted from a depth $l$ down from the surface of the biological tissue; $C_{\mathrm{HbO}_{2}}, C_{\mathrm{Hb}}, C_{\mathrm{MetHb}}$, and $\varepsilon_{\mathrm{HbO}_{2}}(\lambda), \varepsilon_{\mathrm{Hb}}(\lambda), \varepsilon_{\mathrm{MetHb}}(\lambda)$ are the concentrations and molar 


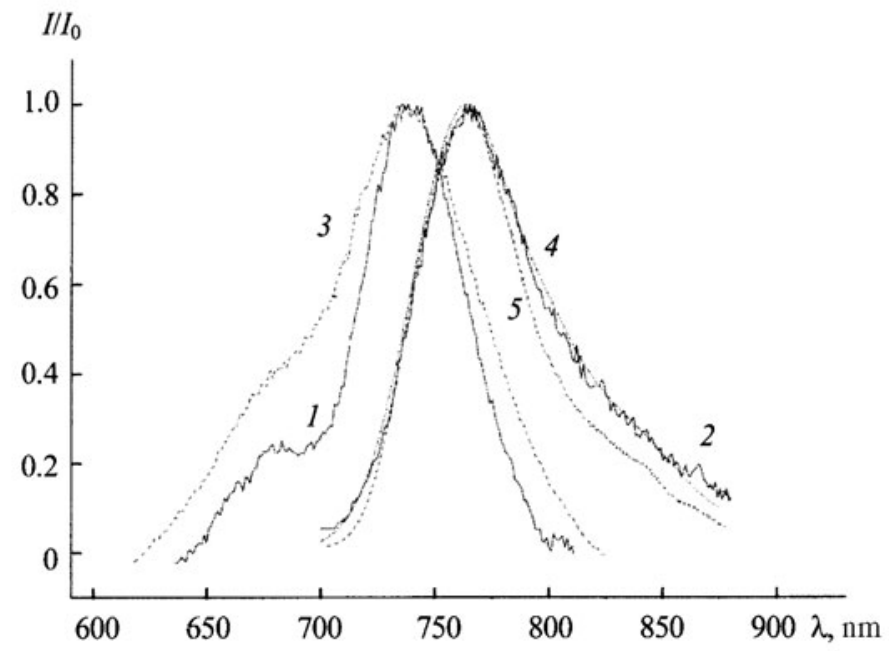

Fig. 5 Absorption spectrum (1) and fluorescence spectrum (2) of PD1 in a culture of HeLa cancer cells, absorption spectrum of PD1 in a sample of liver tissue from which the blood was removed (3), fluorescence spectrum of PD1 in S-45 tumor tissue from which the blood was removed (4), and in a S-45 tumor nodule in vivo (5).

absorption coefficients of $\mathrm{HbO}_{2}, \mathrm{Hb}$, and MetHb respectively. As $I_{0}(\lambda)$, we used the fluorescence spectrum of PD1 in tumor tissue samples from which the blood was removed.

According to available data [8], the scattering coefficient for biological tissues varies insignificantly in the spectral range corresponding to the fluorescence band of PD1, and cannot have a substantial effect on the position and shape of the fluorescence spectrum of PD1. Accordingly, scattering was not considered in this model. In order to calculate the hemoglobin concentration in the rat tumor tissue, we took into account the total blood volume as well as the weight and size of the animal. The hemoglobin concentration in animal blood, according to [15], is $110-120 \mathrm{~g} / \mathrm{L}$. In the calculations, because the signal was averaged over the volume of the samples by using light beams of diameter $\approx 5 \mathrm{~mm}$ on the surface, we assumed that the blood was uniformly distributed over the considered volume. In this case, we took into account a correction for the weight and volume of bone tissue, which does not contain a large amount of blood.

By varying the percent ratio of the $\mathrm{Hb}, \mathrm{HbO}_{2}$, and $\mathrm{MetHb}$ contents according to formula (1), we did a numerical calculation of the fluorescence spectrum of PD1 in vivo, taking into account absorption of the blood components (Fig. 6). As the criterion for correctly choosing the concentrations, we used the condition of least-square deviation of the calculated spectrum from the spectrum recorded in vivo. In this case, the parameters $C_{\mathrm{MetHb}}, C_{\mathrm{Hb}}$, $C_{\mathrm{HbO}_{2}}$, characterizing the concentration of the different forms of hemoglobin, were determined by minimizing the function $\xi\left(C_{\mathrm{MetHb}}, C_{\mathrm{Hb}}, C_{\mathrm{HbO}_{2}}\right)$ by a coordinate descent method [16]:

$$
\xi\left(C_{\mathrm{HbO}_{2}}, C_{\mathrm{Hb}}, C_{\mathrm{MetHb}}\right)=\sum_{\lambda}\left(I(\lambda)-i_{\exp }(\lambda)\right)^{2} .
$$

The value of the function $\xi\left(C_{\mathrm{HbO}_{2}}, C_{\mathrm{Hb}}, C_{\mathrm{MetHb}}\right)$ was determined from formula (2), which represents the square of the difference between the spectrum calculated from formula (1) and the experimentally obtained fluorescence spectrum of the polymethine dye in vivo.

The optimal match between the calculated and experimental spectra of the dye in tumor tissue is obtained for a $\mathrm{HbO}_{2}$ concentration $\mathrm{HbO}_{2}>85 \%$ and an $\mathrm{Hb}$ concentration $\approx 14 \%$ of the total hemoglobin concentration; the MetHb concentration was no greater than $1 \%$. Such a ratio of $\mathrm{HbO}_{2}, \mathrm{Hb}$, and MetHb is consistent with the real concentrations known from the literature for the indicated forms of hemoglobin in rat tissues in vivo [13, 17]. Consequently, a decrease in the half-width of the fluorescence spectrum of the dye in vivo compared with the spectrum in a culture of 


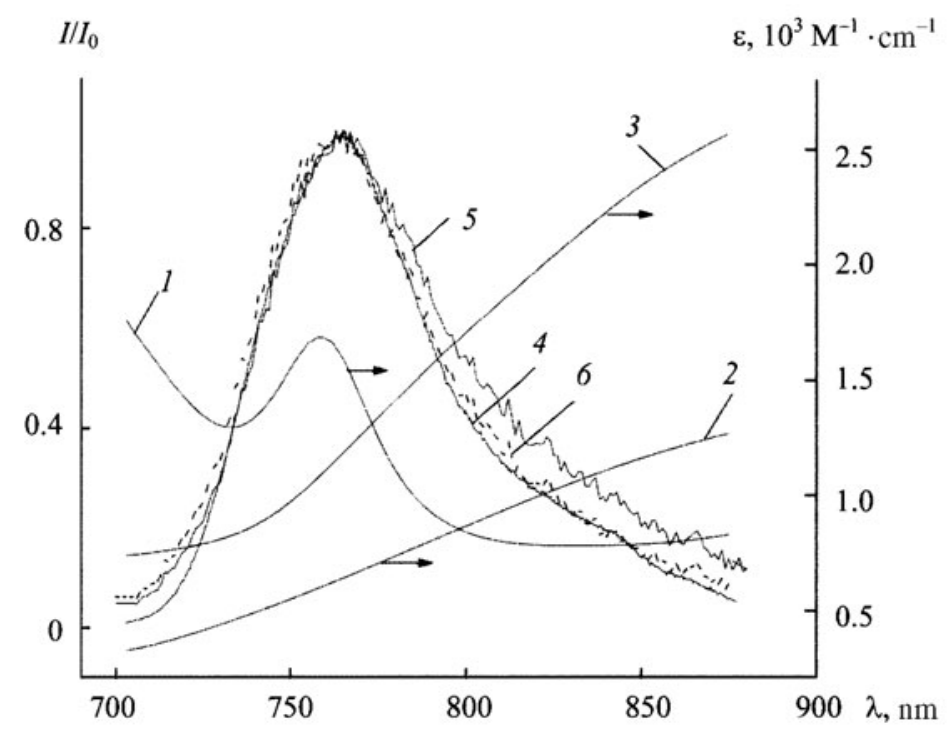

Fig. 6 Absorption spectra of deoxyhemoglobin (1), oxyhemoglobin (2), and methemoglobin (3); fluorescence spectra of PD1 in S-45 tumor nodule in vivo (4), in a culture of HeLa tumor cells (5), and calculated taking into account the effect of absorption by oxyhemoglobin and deoxyhemoglobin (6).

$\mathrm{HeLa}$ tumor cells and in samples of tumor tissue from which the blood has been removed is due mainly to the presence of $\mathrm{HbO}_{2}$ in the animal tissue.

The fluorescence spectra of PD1 in vivo in healthy tissues have a half-width that is slightly smaller and a position of the maximum at shorter wavelengths than in the tumor tissue (Fig. 2). The observed differences can be explained by the effect of the absorption by endogenous biomolecules and the level of oxygenation of the biological tissues. We know [18] that in rapidly growing tumors, which describes sarcoma S-45, a lower oxygen content is observed compared with healthy tissues, i.e., hypoxia. Based on the calculation according to formula (1), we established that a long-wavelength shift of the maximum and broadening of the fluorescence spectrum of PD1 in tumor tissue, compared with normal tissues, are achieved with a 15\%-20\% increase in the Hb fraction in the tumor compared with muscle tissue.

The observed shifts in the absorption and fluorescence spectra of the dyes in HeLa cells and in vivo relative to aqueous and alcohol solutions correlate with changes in the spectra on going to low-polarity organic solvents. Consequently, just as in the cells, the dye molecules in vivo are localized in a medium with low dielectric constant, which is typical for compounds in this class when complexes are formed with proteins or when incorporated into low-polarity regions of the lipid bilayer in cell membranes [19-21]. Since the spectral characteristics of polymethine dyes depend on the polarity of the medium, the observed correspondence allows us to conclude that the PD1 molecules experience practically the same environment in biological tissue and in HeLa tumor cells. Thus the major photophysical properties of the photosensitizer in vivo are sufficiently well modeled when they are studied in a culture of these cancer cells.

Conclusions. During accumulation of the photosensitizer in the living body, the environment of the dye molecules changes according to the extent of redistribution of the photosensitizer from the blood to the tissues. Differences in the fluorescence spectra recorded in normal and tumor tissues are associated with increased deoxyhemoglobin content in the tumor. As a result of comparing the spectral characteristics of polymethine dyes in a culture of $\mathrm{HeLa}$ tumor cells and in the living body, we have established that the dye molecules experience the same environment in the tissue and in the cell culture; in vivo, the dye molecules are also localized in a region with a low dielectric constant of the medium. 


\section{REFERENCES}

1. E. Delaey, A. Kamuhabwa, P. Jacobs, F. Laar, D. Vos, and P. Witte, J. Photochem. Photobiol. B: Biol., 55, 2736 (2000).

2. T. J. Dougherty, C. J. Gomer, B. W. Henderson, G. Jori, D. Kessel, M. Korbelik, J. Moan, and Q. Peng, J. Nat. Cancer Inst., 90, No. 12, 889-905 (1997).

3. Y. P. Istomin, E. N. Alexandrova, E. A. Zhavrid, E. S. Voropay, M. P. Samtsov, K. N. Kaplevsky, A. P. Lugovsky, and A. A. Lugovsky, Exper. Oncology, 28, No. 1, 80-82 (2006).

4. M. P. Samtsov, E. S. Voropai, K. N. Kaplevskii, D. G. Mel'nikov, L. S. Lyashenko, and Yu. P. Istomin, Zh. Prikl. Spektrosk., 76, No. 4, 576-582 (2009).

5. M. P. Samtsov, E. S. Voropai, K. N. Kaplevskii, and D. G. Mel'nikov, Zh. Prikl. Spektrosk., 75, No. 5, 684-693 (2008).

6. A. A. Ishchenko, Structure and Spectral Luminescence Properties of Polymethine Dyes [in Russian], Naukova Dumka, Kiev, Kiev (1994).

7. E. S. Voropai, M. P. Samtsov, K. N. Kaplevskii, A. A. Lugovskii, and E. N. Aleksandrova, Zh. Prikl. Spektrosk., 71, No. 2, 166-172 (2004).

8. P. Taroni, A. Pifferi, A. Torricelli, D. Comelli, and R. Cubeddu, Photochem. Photobiol., 2, 124-129 (2003).

9. I. Wang, "Photodynamic therapy and laser-based diagnostic studies of malignant tumours," Doctoral Thesis, Lund University Medical Laser Centre, Department of Oncology, Lund University Hospital (1999).

10. S. Matcher, C. Elwell, C. Cooper, M. Cope, and D. Delpy, Analyt. Biochem., 227, No. 1, 54-68 (1995).

11. S. Matcher, M. Cope, and D. Delpy, Phys. Med. Biol., 39, 177-196 (1994).

12. W. Zijlstra, A. Buursma, and O. van Assendelft, Visible and Near Infrared Absorption Spectra of Human and Animal Haemoglobin, VSP, Utrecht (2000).

13. O. Abdallah, W. Stork, K. Miller-Glaser, and A. Bolz, Biomedizinische Technik, 48 (2003).

14. E. S. Voropai, M. P. Samtsov, V. N. Chalov, and É. A. Zhavrid, Zh. Prikl. Spektrosk., 68, No. 3, 359-362 (2001).

15. W. Hsing-Wen, E. M. Putt, M. J. Emanuele, D. B. Shin, E. Glatstein, and A. G. Yodh, Cancer Res., 64, No. 20, 7553-61 (2004).

16. A. E. Mudrov, Numerical Methods for Personal Computers in BASIC, FORTRAN, and PASCAL [in Russian], Rasko, Tomsk (1991), pp. 252-257.

17. S. A. Kutsenko, Principles of Toxicology [in Russian], Foliant, St. Petersburg (2004), p. 720.

18. M. Höckel and P. Vaupel, J. Nat. Cancer Inst., 93, No. 4, 266-276 (2001).

19. O. Valdes-Aguilera, L. Cincotta, J. Foley, and I. E. Kochevar, Photochem. Photobiol., 45, No. 3, 337-344 (1987).

20. K. Maruszewski, A. Sidorowicz, A. Pola, and W. Strek, J. Mol. Struct., 444, No. 1-3, 147-153 (1998).

21. A. Sidorowicz, A. Pola, and P. Dobryszycki, J. Photochem. Photobiol., 38, No. 1, 94-97 (1997). 